COMMUNICATIONS IN

ANALYSIS AND GEOMETRY

Volume 12, Number 4, 949-965, 2004

\title{
Convergence of the J-flow on Kähler Surfaces
}

\section{Ben WeINKOVE}

Donaldson defined a parabolic flow of potentials on Kähler manifolds which arises from considering the action of a group of symplectomorphisms on the space of smooth maps between manifolds. One can define a moment map for this action, and then consider the gradient flow of the square of its norm. Chen discovered the same flow from a different viewpoint and called it the $J$-flow, since it corresponds to the gradient flow of his $J$-functional, which is related to Mabuchi's K-energy. In this paper, we show that in the case of Kähler surfaces with two Kähler forms satisfying a certain inequality, the $J$-flow converges to a zero of the moment map.

\section{Introduction.}

In [Do], Donaldson described how a number of geometric situations fit into a general framework of diffeomorphism groups and moment maps. In the Kähler setting, he used this framework to define a natural parabolic flow, as follows. Suppose that $(M, \omega)$ is a compact Kähler manifold of dimension $n$ and let $\chi_{0}$ be another Kähler form on $M$, in a different Kähler class. Consider the infinite-dimensional manifold $\mathcal{M}$ of diffeomorphisms $f: M \rightarrow$ $M$, homotopic to the identity. $\mathcal{M}$ carries a natural symplectic form $\Omega$ defined by

$$
\Omega_{f}(v, w)=\int_{M} \omega(v, w) \frac{\chi_{0}^{n}}{n !},
$$

for sections $v, w$ of $f^{*}(T M)$. The group $\mathcal{G}$ of exact $\chi_{0}$-symplectomorphisms of $M$ acts on $\mathcal{M}$ by composition on the right, preserving $\Omega$. We can identify the Lie algebra of $\mathcal{G}$ with the space of functions on $M$ of integral zero with respect to the volume form induced by $\chi_{0}$. A moment map $\mu: \mathcal{M} \rightarrow \operatorname{Lie}(\mathcal{G})^{*}$ for the group action is given by

$$
\mu(f)=\frac{f^{*}(\omega) \wedge \chi_{0}^{n-1}}{\chi_{0}^{n}}-\frac{\int_{M} \omega \wedge \chi_{0}^{n-1}}{\int_{M} \chi_{0}^{n}},
$$

where we are using the $L^{2}$ inner product to identify $\operatorname{Lie}(\mathcal{G})$ with its dual. It is natural to look for solutions of

$$
\mu(f)=0 \quad(\bmod \mathcal{G}) .
$$


These points form the symplectic quotient. Under certain conditions, one would hope that the gradient flow $f_{t}$ of the function $\|\mu\|^{2}$ on $\mathcal{M}$ would converge to give a solution of (1.1). The gradient flow can be rewritten as a flow of Kähler forms $\left(f_{t}^{*}\right)^{-1}\left(\chi_{0}\right)$ on $M$. This defines a parabolic flow on the space of Kähler potentials and is the object of study of this paper.

At around the same time, Chen [C1] independently discovered the same flow as the gradient flow of his $J$-functional. He later called it the $J$-flow $[\mathrm{C} 2]$. He showed in [C1] that the $J$-functional is related to the Mabuchi K-energy [Ma], which plays a key role in the study of Kähler geometry and stability in the sense of geometric invariant theory (see [Y2], [T2], [T3] and [PS] for example).

Explicitly, the $J$-flow is defined as follows. Let $c$ be the constant given by

$$
c=\frac{\int_{M} \omega \wedge \chi_{0}^{n-1}}{\int_{M} \chi_{0}^{n}},
$$

and let $\mathcal{H}$ be the space of Kähler potentials

$$
\mathcal{H}=\left\{\phi \in C^{\infty}(M) \mid \chi_{\phi}=\chi_{0}+\frac{\sqrt{-1}}{2} \partial \bar{\partial} \phi>0\right\} .
$$

The $J$-flow is the flow on $\mathcal{H}$ given by

$$
\begin{aligned}
\frac{\partial \phi_{t}}{\partial t} & =c-\frac{\omega \wedge \chi_{\phi_{t}}^{n-1}}{\chi_{\phi_{t}}^{n}} . \\
\phi_{0} & =0 .
\end{aligned}
$$

A critical point of the $J$-flow gives a Kähler metric $\chi$ satisfying

$$
\omega \wedge \chi^{n-1}=c \chi^{n} .
$$

Donaldson [Do] asked whether one can find a solution to (1.3) in the class $\left[\chi_{0}\right]$ under certain assumptions. He noted that a necessary condition is that $\left[n c \chi_{0}-\omega\right]$ be a Kähler class, and conjectured that this condition be sufficient. Chen [C1] confirmed this conjecture in the case $n=2$, without using the $J$-flow, by observing that (1.3) reduces to a Monge-Ampère equation which can be solved by the well-known result of Yau [Y1]. The conjecture is still open for $n>2$.

Chen [C1] shows that Donaldson's conjecture would imply a result on the lower bound of the Mabuchi K-energy for compact Kähler manifolds $M$ with negative first Chern class. Namely, if $-\omega \in c_{1}(M)$ with $\omega>0$, then for Kähler classes $\left[\chi_{0}\right]$ satisfying

$$
n c\left[\chi_{0}\right]-[\omega]>0
$$


the Mabuchi K-energy would have a lower bound in the class $\left[\chi_{0}\right]$.

Solutions of the $J$-flow exist for a short time by general theory, since the flow is parabolic. In [C2], Chen showed that the flow always exists for all time for any smooth initial data. He also showed that if the bisectional curvature of $\omega$ is non-negative then the $J$-flow converges to a critical metric.

In general, the behaviour of the flow is not known. In this paper, we deal with the case $n=2$ with no curvature restrictions. Our main result is as follows.

Main Theorem Suppose that $(M, \omega)$ has dimension $n=2$ and that

$$
n c \chi_{0}-\omega>0 \text {. }
$$

Then the J-flow (1.2) converges in $C^{\infty}$ to a smooth critical metric.

The outline of the paper is as follows. In section 2 we state some preliminary facts about the flow and introduce notation. In section 3, the maximum principle is used to derive an estimate on the second derivatives of $\phi$ in terms of $\phi$ itself. In section 4 , a $C^{0}$ estimate for $\phi$ is given. The argument uses the second order estimate, a Moser iteration argument applied to the exponential of $-\phi$ and the result of Tian [T1] (see also [TY]) on the existence of constants $\alpha>0$ and $C$ such that

$$
\int_{M} e^{-\alpha \phi} \frac{\chi_{0}^{n}}{n !} \leq C
$$

for all $\phi$ in $\mathcal{H}$ with $\sup _{M} \phi=0$. In section 5 , the proof of the main theorem is completed.

\section{Preliminaries and notation.}

From now on, assume that $\omega$ has been scaled so that $c=1 / n$. We will work in local coordinates, and write

$$
\omega=\frac{\sqrt{-1}}{2} g_{i \bar{j}} d z^{i} \wedge d z^{\bar{j}}, \quad \chi_{0}=\frac{\sqrt{-1}}{2} \chi_{0 i \bar{j}} d z^{i} \wedge d z^{\bar{j}}
$$

and

$$
\chi=\frac{\sqrt{-1}}{2} \chi_{i \bar{j}} d z^{i} \wedge d z^{\bar{j}}=\frac{\sqrt{-1}}{2}\left(\chi_{0 i \bar{j}}+\partial_{i} \partial_{\bar{j}} \phi\right) d z^{i} \wedge d z^{\bar{j}},
$$

where $\chi=\chi_{\phi}$ (suppressing the $t$-subscript.) The operators $\Lambda_{\omega}$ and $\Lambda_{\chi}$ act on $(1,1)$ forms $\alpha=\frac{\sqrt{-1}}{2} \alpha_{i \bar{j}} d z^{i} \wedge d z^{\bar{j}}$ by

$$
\Lambda_{\omega} \alpha=g^{i \bar{j}} \alpha_{i \bar{j}}, \quad \text { and } \quad \Lambda_{\chi} \alpha=\chi^{i \bar{j}} \alpha_{i \bar{j}} .
$$


The $J$-flow (1.2) can be written

$$
\begin{aligned}
\frac{\partial \phi}{\partial t} & =\frac{1}{n}\left(1-\Lambda_{\chi} \omega\right) \\
\left.\phi\right|_{t=0} & =0 .
\end{aligned}
$$

Differentiating with respect to $t$ gives

$$
\frac{\partial}{\partial t}\left(\frac{\partial \phi}{\partial t}\right)=\tilde{\triangle}\left(\frac{\partial \phi}{\partial t}\right)
$$

where the operator $\tilde{\triangle}$ acts on functions $f$ by

$$
\tilde{\triangle} f=\frac{1}{n} \chi^{k \bar{j}} \chi^{i \bar{l}} g_{i \bar{j}} \partial_{k} \partial_{\bar{l}} f
$$

For convenience, write

$$
h^{k \bar{l}}=\chi^{k \bar{j}} \chi^{i \bar{l}} g_{i \bar{j}}
$$

The tensor $h^{k \bar{l}}$ is positive definite and its inverse defines a Hermitian metric on $M$. The operator $\tilde{\triangle}$ is, up to a constant factor, the Laplacian associated to this Hermitian metric.

By the maximum principle for parabolic equations, (2.2) implies that

$$
\inf _{M}\left(\Lambda_{\chi_{0}} \omega\right) \leq \Lambda_{\chi} \omega \leq \sup _{M}\left(\Lambda_{\chi_{0}} \omega\right)
$$

which gives a lower bound for $\chi$,

$$
\chi \geq \frac{1}{\sup _{M}\left(\Lambda_{\chi_{0}} \omega\right)} \omega .
$$

The $J$-functional [C1] is defined by

$$
J_{\omega, \chi_{0}}(\phi)=\int_{0}^{1} \int_{M} \frac{\partial \phi_{t}}{\partial t} \frac{\omega \wedge \chi_{\phi_{t}}^{n-1}}{(n-1) !} d t
$$

where $\left\{\phi_{t}\right\}$ is a path in $\mathcal{H}$ between 0 and $\phi$. The functional is independent of the choice of path. We will need the following formula for the functional in the case $n=2$. Taking the path $\phi_{t}=t \phi$, we see that

$$
J_{\omega, \chi_{0}}(\phi)=\frac{1}{2} \int_{M} \phi \omega \wedge\left(\chi_{0}+\chi\right)
$$


Chen also makes use of the $I$-functional,

$$
I_{\omega, \chi_{0}}(\phi)=\int_{0}^{1} \int_{M} \frac{\partial \phi_{t}}{\partial t} \frac{\chi_{\phi_{t}}^{n}}{n !} d t
$$

This is a well-known functional in Kähler geometry (see [Ma]). Notice that $I(\phi)=0$ along the flow. For $n=2$, this functional is given by

$$
I_{\omega, \chi_{0}}(\phi)=\frac{1}{6} \int_{M} \phi\left(\chi_{0}^{2}+\chi \wedge \chi_{0}+\chi^{2}\right)
$$

In the course of the paper, $C_{0}, C_{1}, \ldots$ will denote constants depending only on the initial data $\omega$ and $\chi_{0}$. Curvature expressions such as $R_{i \bar{j} k \bar{l}}$ will always refer to the metric $g_{i \bar{j}}$.

\section{Second order estimate.}

We use the maximum principle to obtain an estimate on the second derivative of $\phi$ in terms of $\phi$. We choose to calculate the evolution of $\left(\log \Lambda_{\omega} \chi-A \phi\right)$ for some constant $A$ (compare to [Y1], [Au] or [Si] for the analogous estimate for the well-known Monge-Ampère equation, and [Ca] for the Kähler-Ricci flow.)

Theorem 3.1. Suppose that $(M, \omega)$ has dimension $n=2$ and that

$$
\chi_{0}-\omega>0
$$

Let $\phi=\phi_{t}$ be a solution of the J-flow (2.1) on $[0, \infty)$. Then there exist constants $A>0$ and $C>0$ depending only on the initial data such that for any time $t \geq 0, \chi=\chi_{\phi_{t}}$ satisfies

$$
\Lambda_{\omega} \chi \leq C e^{A\left(\phi-\inf _{M \times[0, t]} \phi\right)} .
$$

Proof. We will calculate

$$
\left(\tilde{\triangle}-\frac{\partial}{\partial t}\right)\left(\log \left(\Lambda_{\omega} \chi\right)-A \phi\right)
$$

Using normal coordinates for $\omega$, first calculate

$$
\begin{aligned}
\tilde{\triangle}\left(\Lambda_{\omega} \chi\right) & =\frac{1}{n} h^{k \bar{l}} \partial_{k} \partial_{\bar{l}}\left(g^{i \bar{j}} \chi_{i \bar{j}}\right) \\
& =\frac{1}{n} h^{k \bar{l}} R_{k \bar{l}}{ }^{i \bar{j}} \chi_{i \bar{j}}+\frac{1}{n} h^{k \bar{l}} g^{i \bar{j}} \partial_{k} \partial_{\bar{l}} \chi_{i \bar{j}} .
\end{aligned}
$$


And

$$
\begin{aligned}
\frac{\partial}{\partial t}\left(\Lambda_{\omega} \chi\right)= & \frac{\partial}{\partial t}\left(g^{i \bar{j}} \partial_{i} \partial_{\bar{j}} \phi\right) \\
= & -\frac{1}{n} g^{i \bar{j}} \partial_{i} \partial_{\bar{j}}\left(\chi^{k \bar{l}} g_{k \bar{l}}\right) \\
= & \frac{1}{n}\left(g^{i \bar{j}} \partial_{i}\left(\chi^{p \bar{l}} \partial_{\bar{j}} \chi_{p \bar{q}} \chi^{k \bar{q}}\right) g_{k \bar{l}}+g^{i \bar{j}} \chi^{k \bar{l}} R_{i \bar{j} k \bar{l}}\right) \\
= & \frac{1}{n}\left(g^{i \bar{j}} h^{p \bar{q}} \partial_{i} \partial_{\bar{j}} \chi_{p \bar{q}}-g^{i \bar{j}} h^{r \bar{q}} \chi^{p \bar{s}} \partial_{i} \chi_{r \bar{s}} \partial_{\bar{j}} \chi_{p \bar{q}}\right. \\
& \left.-g^{i \bar{j}} h^{p \bar{s}} \chi^{r \bar{q}} \partial_{i} \chi_{r \bar{s}} \partial_{\bar{j}} \chi_{p \bar{q}}+\chi^{k \bar{l}} R_{k \bar{l}}\right) .
\end{aligned}
$$

Now

$$
\tilde{\triangle} \log \left(\Lambda_{\omega} \chi\right)=\frac{\tilde{\triangle}\left(\Lambda_{\omega} \chi\right)}{\Lambda_{\omega} \chi}-\frac{\left|\tilde{\nabla}\left(\Lambda_{\omega} \chi\right)\right|^{2}}{\left(\Lambda_{\omega} \chi\right)^{2}}
$$

where

$$
\left|\tilde{\nabla}\left(\Lambda_{\omega} \chi\right)\right|^{2}=\frac{1}{n} h^{k \bar{l}} \partial_{k}\left(\Lambda_{\omega} \chi\right) \partial_{\bar{l}}\left(\Lambda_{\omega} \chi\right)
$$

Note that by the Kähler property of $\chi$, we have

$$
\partial_{i} \partial_{\bar{j}} \chi_{k \bar{l}}=\partial_{k} \partial_{\bar{l}} \chi_{i \bar{j}}
$$

Then

$$
\begin{aligned}
& \left(\tilde{\triangle}-\frac{\partial}{\partial t}\right) \log \left(\Lambda_{\omega} \chi\right) \\
& =\frac{1}{n \Lambda_{\omega} \chi}\left(h^{k \bar{l}} R_{k \bar{l}}{ }^{i \bar{j}} \chi_{i \bar{j}}-n \frac{\left|\tilde{\nabla}\left(\Lambda_{\omega} \chi\right)\right|^{2}}{\Lambda_{\omega} \chi}+g^{i \bar{j}} h^{r \bar{q}} \chi^{p \bar{s}} \partial_{i} \chi_{r \bar{s}} \partial_{\bar{j}} \chi_{p \bar{q}}\right. \\
& \left.\quad+g^{i \bar{j}} h^{p \bar{s}} \chi^{r \bar{q}} \partial_{i} \chi_{r \bar{s}} \partial_{\bar{j}} \chi_{p \bar{q}}-\chi^{k \bar{l}} R_{k \bar{l}}\right) .
\end{aligned}
$$

We need the following lemma to deal with the second term on the right hand side.

\section{Lemma 3.2.}

$$
n\left|\tilde{\nabla}\left(\Lambda_{\omega} \chi\right)\right|^{2} \leq\left(\Lambda_{\omega} \chi\right) g^{i \bar{j}} h^{r \bar{q}} \chi^{p \bar{s}} \partial_{i} \chi_{r \bar{s}} \partial_{\bar{j}} \chi_{p \bar{q}} .
$$


Proof. Using normal coordinates for $\omega$ in which $\chi$ is diagonal, and making use of the Cauchy-Schwartz inequality, we obtain

$$
\begin{aligned}
n\left|\tilde{\nabla}\left(\Lambda_{\omega} \chi\right)\right|^{2} & =\sum_{i, j, k} \chi^{k \bar{k}} \chi^{k \bar{k}} \partial_{k} \chi_{i \bar{i}} \partial_{\bar{k}} \chi_{j \bar{j}} \\
& \leq \sum_{i, j}\left(\sum_{k}\left(\chi^{k \bar{k}}\right)^{2}\left|\partial_{k} \chi_{i \bar{i}}\right|^{2}\right)^{1 / 2}\left(\sum_{k}\left(\chi^{k \bar{k}}\right)^{2}\left|\partial_{k} \chi_{j \bar{j}}\right|^{2}\right)^{1 / 2} \\
& =\left(\sum_{i}\left(\sum_{k}\left(\chi^{k \bar{k}}\right)^{2}\left|\partial_{k} \chi_{i \bar{i}}\right|^{2}\right)^{1 / 2}\right)^{2} \\
& =\left(\sum_{i} \sqrt{\chi_{i \bar{i}}}\left(\sum_{k}\left(\chi^{k \bar{k}}\right)^{2} \chi^{i \bar{i}}\left|\partial_{k} \chi_{i \bar{i}}\right|^{2}\right)^{1 / 2}\right)^{2} \\
& \leq \sum_{i} \chi_{i \bar{i}} \sum_{i, k}\left(\chi^{k \bar{k}}\right)^{2} \chi^{i \bar{i}}\left|\partial_{k} \chi_{i \bar{i}}\right|^{2} \\
& =\left(\Lambda_{\omega} \chi\right) \sum_{i, k}\left(\chi^{k \bar{k}}\right)^{2} \chi^{i \bar{i}} \partial_{k} \chi_{i \bar{i}} \partial_{\bar{k}} \chi_{i \bar{i}} \\
& =\left(\Lambda_{\omega} \chi\right) \sum_{i, k}\left(\chi^{k \bar{k}}\right)^{2} \chi^{i \bar{i}} \partial_{i} \chi_{k \bar{i}} \partial_{\bar{i}} \chi_{i \bar{k}} \\
& \leq\left(\Lambda_{\omega} \chi\right) \sum_{i, j, k}\left(\chi^{k \bar{k}}\right)^{2} \chi^{i \bar{i}} \partial_{j} \chi_{k \bar{i}} \partial_{\bar{j}} \chi_{i \bar{k}} \\
& =\left(\Lambda_{\omega} \chi\right) g^{i \bar{j}} h^{r \bar{q}} \chi^{p \bar{s}} \partial_{i} \chi_{r \bar{s}} \partial_{\bar{j}} \chi_{p \bar{q}} .
\end{aligned}
$$

Let $C_{0}$ be a constant satisfying

$$
R_{k \bar{l}}^{i \bar{j}} \geq-C_{0} g_{k \bar{l}} g^{i \bar{j}} .
$$

Then,

$$
\begin{aligned}
\left(\tilde{\triangle}-\frac{\partial}{\partial t}\right) \log \left(\Lambda_{\omega} \chi\right) & \geq \frac{1}{n \Lambda_{\omega} \chi}\left(-C_{0} h^{k \bar{l}} g_{k \bar{l}} g^{i \bar{j}} \chi_{i \bar{j}}-\chi^{k \bar{l}} R_{k \bar{l}}\right) \\
& =\frac{1}{n}\left(-C_{0} h^{k \bar{l}} g_{k \bar{l}}-\frac{1}{\Lambda_{\omega} \chi} \chi^{k \bar{l}} R_{k \bar{l}}\right) .
\end{aligned}
$$


Now calculate

$$
\begin{aligned}
\left(\tilde{\triangle}-\frac{\partial}{\partial t}\right) \phi & =\frac{1}{n}\left(h^{k \bar{l}} \partial_{k} \partial_{\bar{l}} \phi+\chi^{i \bar{j}} g_{i \bar{j}}-1\right) \\
& =\frac{1}{n}\left(\chi^{k \bar{j}} \chi^{i \bar{l}} g_{i \bar{j}} \chi_{k \bar{l}}-h^{k \bar{l}} \chi_{0 k \bar{l}}+\chi^{i \bar{j}} g_{i \bar{j}}-1\right) \\
& =\frac{1}{n}\left(2 \chi^{i \bar{j}} g_{i \bar{j}}-h^{k \bar{l}} \chi_{0 k \bar{l}}-1\right) .
\end{aligned}
$$

At this point we must choose our value of $A$. From our assumption (3.1), we can choose $0<\epsilon<1 / 3$ to be sufficiently small so that

$$
\chi_{0} \geq(1+3 \epsilon) \omega .
$$

Let $A$ be given by

$$
A=\frac{C_{0}}{\epsilon}
$$

Fix a time $t>0$. There is a point $\left(x_{0}, t_{0}\right)$ in $M \times[0, t]$ at which the maximum of $\left(\log \left(\Lambda_{\omega} \chi\right)-A \phi\right)$ is achieved. We may assume that $t_{0}>0$. At this point, we have

$$
\begin{aligned}
0 \geq & \left(\tilde{\triangle}-\frac{\partial}{\partial t}\right)\left(\log \left(\Lambda_{\omega} \chi\right)-A \phi\right) \\
\geq & \frac{1}{n}\left(-C_{0} h^{k \bar{l}} g_{k \bar{l}}-\frac{1}{\Lambda_{\omega} \chi} \chi^{k \bar{l}} R_{k \bar{l}}-2 A \chi^{i \bar{j}} g_{i \bar{j}}+A h^{k \bar{l}} \chi_{0 k \bar{l}}+A\right) \\
\geq & \frac{1}{n}\left(-C_{0} h^{k \bar{l}} g_{k \bar{l}}-\frac{1}{\Lambda_{\omega} \chi} \chi^{k \bar{l}} R_{k \bar{l}}-2 A \chi^{i \bar{j}} g_{i \bar{j}}+(1-\epsilon) A h^{k \bar{l}} \chi_{0 k \bar{l}}\right. \\
& \left.+\epsilon A h^{k \bar{l}} g_{k \bar{l}}+A\right) \\
= & \frac{1}{n}\left(-\frac{1}{\Lambda_{\omega} \chi} \chi^{k \bar{l}} R_{k \bar{l}}-2 A \chi^{i \bar{j}} g_{i \bar{j}}+(1-\epsilon) A h^{k \bar{l}} \chi_{0 k \bar{l}}+A\right) .
\end{aligned}
$$

From the lower bound (2.4) on $\chi_{k \bar{l}}$, the term $\chi^{k \bar{l}} R_{k \bar{l}}$ is bounded above and hence at $\left(x_{0}, t_{0}\right)$, we have

$$
1+(1-\epsilon) h^{k \bar{l}} \chi_{0 k \bar{l}}-2 \chi^{i \bar{j}} g_{i \bar{j}} \leq \frac{C_{1}}{\left(\Lambda_{\omega} \chi\right)} .
$$

From (3.3), we get

$$
1+(1+\epsilon) h^{k \bar{l}} g_{k \bar{l}}-2 \chi^{i \bar{j}} g_{i \bar{j}} \leq \frac{C_{1}}{\left(\Lambda_{\omega} \chi\right)} .
$$

We will compute in normal coordinates at $x_{0}$ for $\omega$ in which $\chi$ is diagonal and has eigenvalues $\lambda_{1}, \lambda_{2}$. From (2.4), $\lambda_{1}$ and $\lambda_{2}$ are bounded below by 
a positive constant. We want to show that they are also bounded above. First, observe that for $n=2$,

$$
\frac{1}{\Lambda_{\chi} \omega}=\frac{\operatorname{det} \chi}{(\operatorname{det} \omega)\left(\Lambda_{\omega} \chi\right)}
$$

and by (2.3), this is bounded along the flow.

Multiplying (3.4) by $(\operatorname{det} \chi / \operatorname{det} \omega)$ gives,

$$
\lambda_{1} \lambda_{2}+(1+\epsilon)\left(\frac{\lambda_{2}}{\lambda_{1}}+\frac{\lambda_{1}}{\lambda_{2}}\right)-2\left(\lambda_{1}+\lambda_{2}\right) \leq C_{2} .
$$

From (2.3), we may suppose that one of the eigenvalues, say $\lambda_{2}$, is bounded from above. Rewrite the inequality as

$$
\lambda_{1}\left(\lambda_{2}+(1+\epsilon) \frac{1}{\lambda_{2}}-2\right)+(1+\epsilon) \frac{\lambda_{2}}{\lambda_{1}}-2 \lambda_{2} \leq C_{2} .
$$

Then, since the function $f:(0, \infty) \rightarrow \mathbf{R}$ defined by

$$
f(x)=x+(1+\epsilon) \frac{1}{x}-2,
$$

is bounded below by a small positive constant depending on $\epsilon$, we see that $\lambda_{1}$ must also be bounded above. Hence at the point $\left(x_{0}, t_{0}\right)$, there exists $C$ depending only on the initial data such that

$$
\Lambda_{\omega} \chi \leq C
$$

Then, on $M \times[0, t]$,

$$
\log \left(\Lambda_{\omega} \chi\right)-A \phi \leq \log C-A \inf _{M \times[0, t]} \phi .
$$

Exponentiating gives

$$
\Lambda_{\omega} \chi \leq C e^{A\left(\phi-\inf _{M \times[0, t]} \phi\right)},
$$

completing the proof of the theorem.

\section{Zero order estimate.}

We prove an estimate on the $C^{0}$ norm of $\phi$ using a Moser iteration method applied to the exponential of the solution rather than a power of the solution (compare to [Y1]) and the estimate of Theorem 3.1. 
Theorem 4.1. Suppose that $(M, \omega)$ has dimension $n=2$ and that

$$
\chi_{0}-\omega>0 \text {. }
$$

Let $\phi_{t}$ be a solution of the $J$-flow (2.1) on $[0, \infty)$. Then there exists a constant $\tilde{C}$ depending only on the initial data such that

$$
\left\|\phi_{t}\right\|_{C^{0}(M)} \leq \tilde{C}
$$

Proof. Suppose first that $\inf _{M} \phi_{t}$ is bounded from below uniformly in time. We will show that this implies the above estimate. Since the functional $J_{\omega, \chi_{0}}$ decreases along the flow, there exists a constant $C_{0}$ such that

$$
\int_{M} \phi_{t} \omega \wedge\left(\chi_{0}+\chi_{\phi_{t}}\right) \leq C_{0}
$$

using (2.5). Let $C_{1}$ be a positive constant satisfying

$$
\omega^{2} \leq C_{1} \omega \wedge \chi_{0}
$$

Then

$$
\begin{aligned}
\int_{M} \phi_{t} \omega^{2}= & \int_{M}\left(\phi_{t}-\inf _{M} \phi_{t}\right) \omega^{2}+\int_{M} \inf _{M} \phi_{t} \omega^{2} \\
\leq & C_{1} \int_{M}\left(\phi_{t}-\inf _{M} \phi_{t}\right) \omega \wedge \chi_{0}+\inf _{M} \phi_{t} \int_{M} \omega^{2} \\
\leq & C_{1} C_{0}-C_{1} \int_{M} \phi_{t} \omega \wedge \chi_{\phi_{t}}+\inf _{M} \phi_{t}\left(\int_{M} \omega^{2}-C_{1} \int_{M} \omega \wedge \chi_{0}\right) \\
= & C_{1} C_{0}-C_{1} \int_{M}\left(\phi_{t}-\inf _{M} \phi_{t}\right) \omega \wedge \chi_{\phi_{t}} \\
& +\inf _{M} \phi_{t}\left(\int_{M} \omega^{2}-2 C_{1} \int_{M} \omega \wedge \chi_{0}\right) \\
\leq & C_{1} C_{0}+\inf _{M} \phi_{t}\left(\int_{M} \omega^{2}-2 C_{1} \int_{M} \omega \wedge \chi_{0}\right) .
\end{aligned}
$$

This gives an upper bound for $\int_{M} \phi_{t} \omega^{2}$ depending on the lower bound for $\inf _{M} \phi_{t}$. Since $\triangle_{\omega} \phi_{t}>-\Lambda_{\omega} \chi_{0}$ along the flow, it follows from the existence of a lower bound on the Green's function of $\omega$ that $\sup _{M} \phi_{t}$ is bounded from above, giving us the required estimate.

Now suppose that no such lower bound for $\inf _{M} \phi_{t}$ exists. Then we can assume that there is a sequence of times $t_{i} \rightarrow \infty$ such that

(i) $\inf _{M} \phi_{t_{i}}=\inf _{t \in\left[0, t_{i}\right]} \inf _{M} \phi_{t}$ 
(ii) $\inf _{M} \phi_{t_{i}} \rightarrow-\infty$.

We will seek a contradiction. For a fixed $i$, write

$$
\psi_{t_{i}}=\phi_{t_{i}}-\sup _{M} \phi_{t_{i}}
$$

Notice that $\sup _{M} \phi_{t_{i}}$ is bounded from below by zero from (2.6) and the fact that $I\left(\phi_{t}\right)=0$. Hence

$$
\left\|\psi_{t_{i}}\right\|_{C^{0}} \rightarrow \infty \text {. }
$$

The following proposition is the key result of this section.

Proposition 4.2. Let $M$ be a compact complex surface with two Kähler metrics $\chi_{0}$ and $\omega$. Suppose that $\psi \in C^{\infty}(M)$ satisfies the conditions

$$
\chi_{\psi}=\chi_{0}+\frac{\sqrt{-1}}{2} \partial \bar{\partial} \psi>0, \quad \sup _{M} \psi=0,
$$

and

$$
\Lambda_{\omega} \chi_{\psi} \leq C e^{A\left(\psi-\inf _{M} \psi\right)} .
$$

Then there exists a constant $C^{\prime}$ depending only on $M, \omega, \chi_{0}$ and the constants $A$ and $C$ such that

$$
\|\psi\|_{C^{0}} \leq C^{\prime}
$$

We apply this proposition to $\psi=\psi_{t_{i}}$ and obtain a contradiction since

$$
\begin{aligned}
\Lambda_{\omega} \chi_{\psi_{t_{i}}} & =\Lambda_{\omega} \chi_{\phi_{t_{i}}} \\
& \leq C e^{A\left(\phi_{t_{i}}-\inf _{t \in\left[0, t_{i}\right]} \inf _{M} \phi_{t}\right)} \\
& =C e^{A\left(\psi_{t_{i}}-\inf _{M} \psi_{t_{i}}\right)},
\end{aligned}
$$

where we have used Theorem 3.1 and condition (i) above. It remains to prove the proposition.

Proof of Proposition 4.2 Let $\delta$ be a small positive constant, to be determined later. Set $B=A /(1-\delta)$ and let $u=e^{-B \psi}$.

Now, for $\beta=n /(n-1)=2$, the Sobolev inequality for functions $f$ on $(M, \omega)$ is

$$
\|f\|_{2 \beta}^{2} \leq C_{2}\left(\|\nabla f\|_{2}^{2}+\|f\|_{2}^{2}\right),
$$

for $C_{2}$ depending on $\omega$. We will apply this to $u^{p / 2}$ for $p \geq 1$. This gives

$$
\left(\int_{M} e^{-B p \beta \psi} \frac{\omega^{2}}{2}\right)^{1 / \beta} \leq C_{2}\left(\int_{M}\left|\nabla e^{-B p \psi / 2}\right|^{2} \frac{\omega^{2}}{2}+\int_{M} e^{-B p \psi} \frac{\omega^{2}}{2}\right) .
$$


Now calculate

$$
\begin{aligned}
\int_{M}\left|\nabla e^{-B p \psi / 2}\right|^{2} \frac{\omega^{2}}{2} & =\sqrt{-1} \int_{M} \partial e^{-B p \psi / 2} \wedge \bar{\partial} e^{-B p \psi / 2} \wedge \omega \\
& =\frac{B^{2} p^{2}}{4} \sqrt{-1} \int_{M} e^{-B p \psi} \partial \psi \wedge \bar{\partial} \psi \wedge \omega \\
& =-\frac{B p}{4} \sqrt{-1} \int_{M} \partial\left(e^{-B p \psi}\right) \wedge \bar{\partial} \psi \wedge \omega \\
& =\frac{B p}{2} \int_{M} e^{-B p \psi} \frac{\sqrt{-1}}{2} \partial \bar{\partial} \psi \wedge \omega \\
& =\frac{B p}{2} \int_{M} e^{-B p \psi}\left(\chi_{\psi}-\chi_{0}\right) \wedge \omega \\
& =\frac{B p}{2} \int_{M} e^{-B p \psi}\left(\Lambda_{\omega} \chi_{\psi}-\Lambda_{\omega} \chi_{0}\right) \frac{\omega^{2}}{2} \\
& \leq \frac{C B p}{2} \int_{M} e^{-B p \psi} e^{A\left(\psi-\inf _{M} \psi\right)} \frac{\omega^{2}}{2} \\
& =\frac{C B p}{2} e^{-A \inf _{M} \psi} \int_{M} e^{-(p-(1-\delta)) B \psi} \frac{\omega^{2}}{2}
\end{aligned}
$$

where we have used the estimate

$$
\Lambda_{\omega} \chi_{\psi} \leq C e^{A\left(\psi-\inf _{M} \psi\right)} .
$$

Then in (4.1),

$$
\left(\int_{M} u^{p \beta} \frac{\omega^{2}}{2}\right)^{1 / \beta} \leq C_{3} p e^{-A \inf _{M} \psi} \int_{M} u^{p-(1-\delta)} \frac{\omega^{2}}{2} .
$$

Raising to the power $1 / p$ and writing $\gamma=1-\delta$ gives

$$
\|u\|_{p \beta} \leq C_{3}^{1 / p} p^{1 / p} e^{-(A / p) \inf _{M} \psi}\|u\|_{p-\gamma}^{(p-\gamma) / p} .
$$

Take the logarithm of both sides to get

$$
\log \|u\|_{p \beta} \leq \frac{1}{p} \log C_{3}+\frac{1}{p} \log p+\frac{1}{p} \sup _{M}(-A \psi)+\frac{(p-\gamma)}{p} \log \|u\|_{p-\gamma} .
$$

We now apply the iteration. First, replace $p$ with $p \beta+\gamma$ to get

$$
\begin{aligned}
\log \|u\|_{p \beta^{2}+\gamma \beta} \leq & \frac{1+\beta}{p \beta+\gamma} \log C_{3}+\frac{1}{p \beta+\gamma}(\beta \log p+\log (p \beta+\gamma)) \\
& +\frac{1+\beta}{p \beta+\gamma} \sup _{M}(-A \psi)+\frac{\beta(p-\gamma)}{p \beta+\gamma} \log \|u\|_{p-\gamma} .
\end{aligned}
$$


Repeat this procedure, replacing $p$ with $p \beta+\gamma$ to obtain for any positive integer $k$,

$$
\begin{aligned}
\log \|u\|_{p \beta^{k+1}+\gamma\left(\beta+\beta^{2}+\ldots+\beta^{k}\right)} & \\
\leq & \frac{1+\beta+\beta^{2}+\ldots+\beta^{k}}{p \beta^{k}+\gamma\left(1+\beta+\beta^{2}+\ldots+\beta^{k-1}\right)} \log C_{3} \\
& +\frac{1}{p \beta^{k}+\gamma\left(1+\beta+\ldots+\beta^{k-1}\right)}\left(\beta^{k} \log p+\beta^{k-1} \log (p \beta+\gamma)+\ldots\right. \\
& \ldots+\log \left(p \beta^{k}+\gamma\left(1+\beta+\ldots+\beta^{k-1}\right)\right) \\
& +\frac{1+\beta+\beta^{2}+\ldots+\beta^{k}}{p \beta^{k}+\gamma\left(1+\beta+\beta^{2}+\ldots+\beta^{k-1}\right)} \sup (-A \psi) \\
& +\frac{\beta^{k}(p-\gamma)}{p \beta^{k}+\gamma\left(1+\beta+\beta^{2}+\ldots+\beta^{k-1}\right)} \log \|u\|_{p-\gamma} .
\end{aligned}
$$

Now set $p=1+\delta$. Then, since $\beta=2$ we have

$$
p \beta^{k}+\gamma\left(1+\beta+\beta^{2}+\ldots+\beta^{k-1}\right)=1+\beta+\beta^{2}+\ldots+\beta^{k}+\delta .
$$

Notice that the second term on the right hand side of (4.2) is bounded by

$$
\begin{aligned}
\log p+\frac{1}{\beta} \log \beta^{2}+\ldots+\frac{1}{\beta^{k}} \log \left(\beta^{k+1}\right) & \leq \log p+\log \beta\left(\sum_{i=1}^{k} \frac{i+1}{\beta^{i}}\right) \\
& \leq C_{4} .
\end{aligned}
$$

Then

$$
\begin{array}{r}
\log \|u\|_{p \beta^{k+1}+\gamma\left(\beta+\beta^{2}+\ldots+\beta^{k}\right)} \\
\leq \log C_{3}+C_{4}+\sup _{M}(-A \psi)+2 \delta \max \left(\log \|u\|_{2 \delta}, 0\right) .
\end{array}
$$

Using the fact that $A=(1-\delta) B$ and $-B \psi=\log u$, and letting $k$ tend to infinity,

$$
\log \|u\|_{C_{0}} \leq C_{5}+2 \max \left(\log \|u\|_{2 \delta}, 0\right) .
$$

Hence we get the following inequality for $\psi$,

$$
\|\psi\|_{C^{0}} \leq C_{6}+C_{7} \max \left(\log \left(\int_{M} e^{-2 \delta B \psi} \frac{\omega^{2}}{2}\right)^{1 / 2 \delta}, 0\right) .
$$

We can now finish the estimate. First, define

$$
P\left(M, \chi_{0}\right)=\left\{\Phi \in C^{2}(M) \mid \chi_{0}+\frac{\sqrt{-1}}{2} \partial \bar{\partial} \Phi \geq 0, \sup _{M} \Phi=0\right\} .
$$


Then Proposition 2.1 of [T1] (see section 4.4, [Ho]) states that there exist constants $\alpha>0$ and $C_{8}$ depending only on $\left(M, \chi_{0}\right)$ such that

$$
\int_{M} e^{-\alpha \Phi} \frac{\chi_{0}^{n}}{n !} \leq C_{8} \quad \text { for all } \Phi \in P\left(M, \chi_{0}\right)
$$

Define $\delta$ to be

$$
\delta=\min \left\{\frac{\alpha}{4 A}, \frac{1}{2}\right\}>0 .
$$

Then the required estimate follows from (4.3), since $\psi$ belongs to $P\left(M, \chi_{0}\right)$.

\section{Convergence of the flow.}

In this section we complete the proof of the main theorem. We assume, using the result of $[\mathrm{C} 2]$, that a solution $\phi=\phi_{t}$ for the $J$-flow exists for all time. From Theorem 3.1 and Theorem 4.1 we have uniform estimates on $\phi$ and the derivatives $\partial_{i} \partial_{\bar{j}} \phi$, using the fact that

$$
\chi_{i \bar{j}}=\chi_{0 i \bar{j}}+\partial_{i} \partial_{\bar{j}} \phi>0 .
$$

Since the operator

$$
\frac{1}{n}\left(1-\Lambda_{\chi} \omega\right)
$$

is concave in the $\chi_{i \bar{j}}$, it is well known that, by the work of Evans [E1, E2] and Krylov [Kr] (see also [Tr]), one can deduce a uniform Hölder estimate on the second derivatives $\partial_{i} \partial_{\bar{j}} \phi$. By differentiating the equation (2.1) and applying standard Schauder estimates for parabolic equations (see [LSU] for example), one can obtain uniform estimates on all of the derivatives of $\phi$. It then follows that there is a sequence of times $t_{j} \rightarrow \infty$ such that $\phi_{t_{j}}$ converges in $C^{\infty}$ to some smooth function $\phi_{\infty}$. In order to show that we have convergence without having to pass to a subsequence, we will use a modification of the argument in $[\mathrm{Ca}]$.

Notice that $\partial \phi / \partial t$ satisfies the heat equation

$$
\frac{\partial}{\partial t}\left(\frac{\partial \phi}{\partial t}\right)=\tilde{\triangle}\left(\frac{\partial \phi}{\partial t}\right)
$$

Since we have uniform bounds for $\chi_{i \bar{j}}$ from above and away from zero, and

bounds on $\frac{\partial}{\partial t} \chi_{i \bar{j}}$ and all the covariant derivatives of $\chi_{i \bar{j}}$ and $\frac{\partial}{\partial t} \chi_{i \bar{j}}$, it follows from the Harnack inequality of Li and Yau [LY] and the argument in [Ca] 
that there exist positive constants $C_{0}$ and $\eta$, which are independent of $t$, such that

$$
\sup _{M}\left(\frac{\partial \phi}{\partial t}\right)-\inf _{M}\left(\frac{\partial \phi}{\partial t}\right) \leq C_{0} e^{-\eta t}
$$

Since

$$
\int_{M} \frac{\partial \phi}{\partial t} \chi^{2}=0
$$

$\partial \phi / \partial t$ must take on the value zero somewhere on $M$ for each $t$, and so

$$
\left|\frac{\partial \phi}{\partial t}\right| \leq C_{0} e^{-\eta t}
$$

Hence for any $0<s<s^{\prime}$, and any $x \in M$,

$$
\begin{aligned}
\left|\phi\left(x, s^{\prime}\right)-\phi(x, s)\right| & =\left|\int_{s}^{s^{\prime}} \frac{\partial \phi}{\partial t}(x, t) d t\right| \\
& \leq \int_{s}^{s^{\prime}}\left|\frac{\partial \phi}{\partial t}(x, t)\right| d t \\
& \leq C_{0} \int_{s}^{s^{\prime}} e^{-\eta t} d t \\
& =C_{0} \frac{1}{\eta}\left(e^{-\eta s}-e^{-\eta s^{\prime}}\right),
\end{aligned}
$$

which tends to zero as $s$ and $s^{\prime}$ tend to infinity. Hence $\phi_{t}$ converges in the $C_{0}$ norm to $\phi_{\infty}$. It must converge also in the $C^{\infty}$ topology, since otherwise there would exist an integer $N$, an $\epsilon>0$ and a sequence $t_{j} \rightarrow \infty$ with

$$
\left\|\phi_{t_{j}}-\phi_{\infty}\right\|_{C^{N}} \geq \epsilon
$$

Since $\phi$ is bounded in all the $C^{k}$ norms, one could pass to a subsequence of the $\phi_{t_{j}}$ which would converge to some $\phi_{\infty}^{\prime} \neq \phi_{\infty}$, giving the contradiction. This completes the proof.

Acknowledgements. This work was completed while the author was a graduate student at Columbia University, and these results form part of his $\mathrm{PhD}$ thesis [We]. The author is very grateful to his advisor D.H. Phong for his constant support and advice. He also thanks Jacob Sturm and Jian Song for some helpful conversations, and the referee for some constructive comments. 


\section{References.}

[Au] Aubin, T. Equations du type Monge-Ampère sur les variétés Kähleriennes compacts, Bull. Sc. Math. 102 (1978), 119-121

[Ca] Cao, H-D. Deformation of Kähler metrics to Kähler-Einstein metrics on compact Kähler manifolds, Invent. Math. 81 (1985), 359-372

[C1] Chen, X. X. On the lower bound of the Mabuchi energy and its application, Int. Math. Res. Notices 12 (2000), 607-623

[C2] Chen, X. X. A new parabolic flow in Kähler manifolds, preprint, arXiv: math.DG/0009247

[Do] Donaldson, S. K. Moment maps and diffeomorphisms, Asian J. Math. 3, No. 1 (1999), 1-16

[E1] Evans, L. C. Classical solutions of fully nonlinear, convex, second order elliptic equations, Comm. Pure Appl. Math. 25 (1982), 333-363

[E2] Evans, L. C. Classical solutions of the Hamilton-Jacobi Bellman equation for uniformly elliptic operators, Trans. Amer. Math. Soc. 275 (1983), 245-255

[Ho] Hörmander, L. An introduction to complex analysis in several variables, Van Nostrand, Princeton, NJ 1973

[Kr] Krylov, N. V. Boundedly nonhomogeneous elliptic and parabolic equations, Izvestia Akad. Nauk. SSSR 46 (1982), 487-523. English translation in Math. USSR Izv. 20 (1983), No. 3, 459-492

[LSU] Ladyzenskaja, O. A., Solonnikov, V. A. and Ural'Ceva, N. N. Linear and quasilinear equations of parabolic type, Providence, Amer. Math. Soc. 1968

[LY] Li, P. and Yau, S.-T. On the parabolic kernel of the Schrödinger operator, Acta Math. 156 (1986), No. 3-4, 153-201

[Ma] Mabuchi, T. K-energy maps integrating Futaki invariants, Tôhoku Math. Journ., 38 (1986), 575-593

[PS] Phong, D. H. and Sturm, J. Stability, energy functionals, and KählerEinstein metrics, Comm. Anal. Geom. 11 (2003), No. 3, 565-597 
[Si] Siu, Y.-T. Lectures on Hermitian-Einstein metrics for stable bundles and Kähler-Einstein metrics, Birkhäuser Verlag, Basel 1987

[T1] Tian, G. On Kähler-Einstein metrics on certain Kähler manifolds with $c_{1}(M)>0$, Invent. Math. 89 (1987), 225-246

[T2] Tian, G. The K-energy on hypersurfaces and stability, Comm. Anal. Geom. 2 (1994), No. 2, 239-265

[T3] Tian, G. Kähler-Einstein metrics with positive scalar curvature, Invent. math. 137 (1997), 1-37

[TY] Tian, G. and Yau, S.-T. Kähler-Einstein metrics on complex surfaces with $c_{1}(M)$ positive, Comm. Math. Phys. 112 (1987),

[Tr] Trudinger, N. S. Fully nonlinear, uniformly elliptic equations under natural structure conditions, Trans. Amer. Math. Soc. 278 (1983), 751-769

[We] Weinkove, B. The J-flow, the Mabuchi energy, the Yang-Mills flow and multiplier ideal sheaves, $\mathrm{PhD}$ thesis, Columbia University 2004

[Y1] Yau, S.-T. On the Ricci curvature of a compact Kähler manifold and the complex Monge-Ampère equation, I, Comm. Pure Appl. Math. 31 (1978), 339-411

[Y2] Yau, S.-T. Open problems in geometry, Proc. Symposia Pure Math. 54 (1993), 1-28 (problem 65)

Department of Mathematics

COlumbia University

NEW YoRK, NY 10027

weinkove@math. columbia.edu

Received August 12, 2003. 